\title{
Modular Properties of the Hard Hexagon Model
}

\author{
Craig A. Tracy, ${ }^{1}$ Larry Grove, ${ }^{2}$ and M. F. Newman ${ }^{2,3}$
}

Received July 22, 1986; revision received April 14, 1987

The physical quantities (or powers thereof) in the hard-hexagon model that were computed exactly by Baxter are shown to be modular functions with respect to the number-theoretic group $\Gamma_{1}[N]$. This allows us to determine the analytic structure of $\kappa$, the partition function per site in the thermodynamic limit, and $\rho$, the density, as functions of the activity $z$.

KEY WORDS: Exactly solvable models; hard hexagon model; modular functions.

\section{INTRODUCTION}

The hard hexagon model has been solved exactly by Baxter ${ }^{(13)}$ (see also Baxter and Pearce ${ }^{(4-6)}$ ). For reviews of the hard hexagon model see Baxter $^{(7)}$ and Pearce. ${ }^{(8)}$ Baxter gives explicit formulas for $\kappa$, the partition function per site in the thermodynamic limit, the density $\rho$, and the activity $z$ as functions of a point $\tau$ in the upper half-plane $\mathscr{H}$. It is the purpose of this paper to show that these physical quantities (or powers thereof) are modular functions with respect to certain number-theoretic groups and then to use the known mathematical theory of modular functions to determine the analytic properties of $\kappa(z)$ and $\rho(z)$ in the complex activity plane.

For the disordered regime, we show that in the finite $z$ plane, $\kappa(z)$ and $\rho(z)$ have on the physical sheet branch points only at the critical activity $z_{c}$ and at a nonphysical activity $z_{\mathrm{NP}}<0$ with $\left|z_{\mathrm{NP}}\right|<z_{c}$. The analytic continuation of $\kappa(z)$ and $\rho(z)$ gives a 24-sheeted covering of the $z$ plane. The only possible branch points occur at $z=0, z_{c}, z_{\mathrm{NP}}$, and $\infty$, with $z=0$ being a holomorphic point on the physical sheet. The explicit behavior of

\footnotetext{
${ }^{1}$ Department of Mathematics, University of California, Davis, California 95616.

${ }^{2}$ Department of Mathematics, University of Arizona, Tucson, Arizona 85721.

${ }^{3}$ Permanent Address: Maths, IAS, ANU, Canberra ACT 2601, Australia.
} 
$\kappa(z)$ and $\rho(z)$ on the physical sheet in the neighborhood of $z_{c}$ and $z_{\mathrm{NP}}$ is given below in (4.3), (4.8), (4.9), and (4.11). This structure on the physical sheet appears to be the simplest structure possible consistent with the fact that there is a phase transition and general theorems concerning cluster expansions. ${ }^{(9)}$ Furthermore, we remark that this analytic structure on the physical sheet is precisely that predicted by Gaunt ${ }^{(10)}$ on the basis of a Padé analysis of series expansions.

The key observation for this analysis is that the physical quantities (or powers of these quantities in certain cases) are modular functions. The groups that arise are $\Gamma_{1}[N]$ for $N=5,15,30$, and 45 , where

$$
\begin{aligned}
\Gamma_{1}[N]= & \left\{A=\left(\begin{array}{ll}
a & b \\
c & d
\end{array}\right) \mid a, b, c, d\right. \text { integers, } \\
& \left.a d-b c=1, \quad\left(\begin{array}{ll}
a & b \\
c & d
\end{array}\right) \equiv \pm\left(\begin{array}{ll}
1 & * \\
0 & 1
\end{array}\right) \bmod N\right\}
\end{aligned}
$$

This is discussed in Section 2. The activity function $z(\tau)$ is closely related to the Klein icosahedron function $\zeta(\tau)$ and plays a distinguished role in our analysis, as discussed in Section 3. In Section 4 the analytic structure of $\kappa$ and $\rho$ in the complex activity plane is discussed. Many aspects of this paper are rather technical and have therefore been relegated to the Appendices. In Appendix A some well-known properties of the Dedekind functions are summarized for the convenience of the reader. Our main reference here is Schoeneberg. ${ }^{(11)}$ Some elementary properties of the groups $\Gamma_{1}[N]$ and tables of local uniformizing variables are given in Appendix B. Also in Appendix B one finds tables that give the local expansions of the physical quantities at various cusps. Complete tables are given for the disordered regime, and for the ordered regime enough information is given for an analysis on the physical sheet. To prove Theorem 2.1, the generators of the groups $\Gamma_{1}[N], N=5,15,30$, and 45 , are needed. Lists of generators are given in Appendix $\mathrm{C}$ along with the method used to find these generators. We could not find these results in the literature. Further information can be extracted from these tables than what is given in Section 4 .

Finally, we mention that it follows from our results and general theorems from Riemann surface theory that in the disordered regime the equation of state can be given implicitly by

$$
P(\kappa, \rho)=0
$$

where $P(x, y)$ is a polynomial in two variables. We show that the valence of $\kappa$ is 22 and the valence of $\rho$ is 8 , from which it follows that there exists a 
$P$ of degree less than or equal to 8 in $\kappa$ and of degree less than or equal to 22 in $\rho$. The explicit determination of $P$ is not done here. In the ordered regime, there will exist, for example, polynomial relations of the form

$$
P\left(\kappa^{3}, R^{3}\right)=0, \quad P\left(\kappa^{3}, \rho_{2}\right)=0
$$

(different $P$, of course), where $R$ is the order parameter $\rho_{1}-\rho_{2}$ and $\rho_{i}$ is the sublattice density. ${ }^{(1-3)}$

\section{HARD HEXAGON MODEL}

If $\mathscr{H}=\{z \in \mathbb{C} \mid \operatorname{Im}(z)>0\}$ denotes the upper half-plane, then Baxter ${ }^{(1-3)}$ has shown that in the disordered regime

$$
\begin{aligned}
& \kappa(\tau)=\frac{\eta^{2}(5 \tau)}{\eta^{2}(6 \tau)} \frac{\eta_{(1,0)}^{2}(5 \tau ; 5) \eta_{(3,0)}(6 \tau ; 6) \eta_{(2,0)}(6 \tau ; 6)}{\eta_{(2,0)}^{3}(5 \tau ; 5) \eta_{(1,0)}(6 \tau ; 6)} \\
& z(\tau)=-\left[\frac{\eta_{(1,0)}(5 \tau ; 5)}{\eta_{(2,0)}(5 \tau ; 5)}\right]^{5} \\
& \rho(\tau)=-\frac{\eta(2 \tau) \eta(3 \tau)}{\eta(\tau) \eta(6 \tau)}\left[\eta_{(1,0)}(5 \tau ; 5) \eta_{(2,0)}(30 \tau ; 5)\right]^{-1}
\end{aligned}
$$

where the physical values of $\tau \in \mathscr{H}$ are $\operatorname{Re} \tau=1 / 2$, and in the ordered regime

$$
\begin{aligned}
\kappa(\tau) & =\frac{\eta^{2}(5 \tau)}{\eta^{2}(3 \tau)} \frac{\eta_{(2,0)}^{2}(5 \tau ; 5) \eta_{(1,0)}(3 \tau ; 3)}{\eta_{(1,0)}^{3}(5 \tau ; 5)} \\
z(\tau) & =\left[\frac{\eta_{(2,0)}(5 \tau ; 5)}{\eta_{(1,0)}(5 \tau ; 5)}\right]^{5} \\
R(\tau) & =\rho_{1}-\rho_{2}=\frac{\eta(\tau) \eta(5 \tau)}{\eta^{2}(3 \tau)} \\
\rho_{2}(\tau) & =\rho_{3}(\tau)=\frac{\eta(\tau) \eta(9 \tau)}{\eta^{2}(3 \tau)}\left[\eta_{(2,0)}(5 \tau ; 5) \eta_{(2,0)}(45 \tau ; 5)\right]^{-1}
\end{aligned}
$$

and the physical values of $\tau \in \mathscr{H}$ in the ordered regime are $\operatorname{Re} \tau=0$.

We have written Baxter's results in terms of the Dedekind eta function $\eta(\tau)$ and the generalized Dedekind function $\eta_{\mathrm{g}}(\tau ; N)$ (see Schoeneberg ${ }^{(11)}$ and Appendix A), as this representation is convenient for the cusp analysis below. The function $\eta^{24}(\tau)$ is a cusp form of weight 12 for the modular group, and the Dedekind functions $\eta_{\mathrm{g}}(\tau ; N), g \neq(0,0) \bmod N$, raised to sufficiently high powers, are modular functions of level $N$. Our first result 
shows that the physical quantities (2.1)-(2.7) (or powers thereof) are modular functions.

Theorem 2.1. In the disordered regime, $z(\tau)$ is a modular function with respect to the group $\Gamma_{1}[5]$, and $\kappa(\tau)$ and $\rho(\tau)$ are modular functions with respect to the group $\Gamma_{1}[30]$. In the ordered regime, $z(\tau)$ is a modular function with respect to the group $\Gamma_{1}[5], \kappa^{3}(\tau)$ and $R^{3}(\tau)$ are modular functions with respect to the group $\Gamma_{1}[15]$, and $\rho_{2}(\tau)=\rho_{3}(\tau)$ are modular functions with respect to the group $\Gamma_{1}[45]$.

Proof. First observe that if we let

$$
\tau \rightarrow \frac{a \tau+b}{c \tau+d}, \quad\left(\begin{array}{ll}
a & b \\
c & d
\end{array}\right) \in S L(2, \mathbb{Z})
$$

then in the quantities (2.1)-(2.7) we encounter functions of the form $f(n \tau)$. In these we use the identity

$$
n \frac{a \tau+b}{c \tau+d}=\frac{a(n \tau)+n b}{(c / n)(n \tau)+d}
$$

Thus, if $n \mid c$,

$$
\left(\begin{array}{cc}
a & n b \\
c / n & d
\end{array}\right) \in S L(2, \mathbb{Z})
$$

and this gives a new element in $S L(2, \mathbb{Z})$. Now let $f$ denote any of the quantities (2.1)-(2.7) and $N$ the corresponding integer in $\Gamma_{1}[N]$ in the statement of the theorem. Using the above observation, the representation of the various physical quantities in terms of $\eta(\tau)$ and $\eta_{\mathrm{g}}(\tau ; N)$, and the transformation properties (A.3) and (A.4) (see Appendix A), we see that

$$
f\left(\frac{a \tau+b}{c \tau+d}\right)=\exp [\pi i r(A)] f(\tau), \quad r(A) \in \mathbb{Q}
$$

for

$$
A=\left(\begin{array}{ll}
a & b \\
c & d
\end{array}\right) \in \Gamma_{1}[N]
$$

The period $r(A)$ can be expressed (using the formulas in Appendix A) in terms of various Dedekind sums. This expression is quite complicatedlooking, but it satisfies

$$
r(A B)=r(A)+r(B)+\text { even integer }
$$


For any particular choice of $A \in \Gamma_{1}[N], r(A)$ can be evaluated by, say, computer algebra means. In Appendix $C$, lists of generators for the groups $\Gamma_{1}[N], N=5,15,30,45$, are given. The periods $r(A)$ are evaluated for each generator, with the result that $\exp [\pi \operatorname{ir}(A)]=1$ for every $A$ for quantities (2.1)-(2.3), (2.5), and (2.7) and $\exp [\pi \operatorname{ir}(A)]$ is a cube root of unity for quantities (2.4) and (2.6). Finally, $f$ is holomorphic for $\tau \in \mathscr{H}$ and is meromorphic at the cusps. This follows from the corresponding properties of $\eta(\tau)$ and $\eta_{\mathrm{g}}(\tau ; N)$.

\section{KLEIN'S ICOSAHEDRON FUNCTION AND THE ACTIVITY FUNCTION}

The Klein icosahedron function ${ }^{(12.13)}$

$$
\begin{aligned}
\zeta(\tau) & =\frac{\eta_{(1,0)}(5 \tau ; 5)}{\eta_{(2,0)}(5 \tau ; 5)} \\
& =e^{2 \pi i \tau / 5} \frac{\sum_{n=-\infty}^{\infty}(-1)^{n} e^{5 \pi i n^{2} \tau+3 \pi i n \tau}}{\sum_{n=-\infty}^{\infty}(-1)^{n} e^{5 \pi i n^{2} \tau+\pi i n \tau}}
\end{aligned}
$$

is a modular function on $\Gamma[5]$. In fact, every modular function on $\Gamma[5]$ is a rational function of $\zeta(\tau)$; in particular, the absolute modular invariant function $J(\tau)$ and $\zeta(\tau)$ are related by the icosahedron equation ${ }^{(12,13)}$

$$
J(\tau)=-u^{3}(\zeta) / 1728 w(\zeta)^{5}
$$

where the polynomials $u$ and $w$ are

$$
\begin{aligned}
& u(x)=x^{20}+1-228\left(x^{15}-x^{5}\right)+494 x^{10} \\
& w(x)=x\left(x^{10}+11 x^{5}-1\right)
\end{aligned}
$$

The icosahedron function $\zeta(\tau)$ is an analytic bijection from the compact Riemann surface $\hat{\mathscr{H}} / \Gamma[5]$ onto $\hat{\mathbb{C}}=\mathbb{C} \cup\{\infty\}$, and the modular invariant $J$ is an analytic bijection from $\hat{\mathscr{H}} / S L(2, \mathbb{Z})$ onto $\widehat{\mathbb{C}}{ }^{(11)}$ Note that $J$ is holomorphic for $\tau \in \mathscr{H}$ and $J(i \infty)=\infty$.

Thus, we see that the activity is

$$
z(\tau)= \begin{cases}-\zeta^{5}(\tau) & \text { disordered regime } \\ \zeta^{-5}(\tau) & \text { ordered regime }\end{cases}
$$


and the modular invariant $J$ in terms of the activity $\%$ is

$$
J=-\frac{\left[z^{4}+1+228\left(z^{3}-z\right)+494 z^{2}\right]^{3}}{1728 z\left(z^{2}-11 z-1\right)^{5}}
$$

It follows from this last expression that $J=\infty$ at $z=0, \infty, z_{c}, z_{\mathrm{NP}}$, where

$$
\begin{aligned}
& z_{c}=\frac{11+5 \sqrt{5}}{2}=\left(\frac{\sqrt{5}+1}{2}\right)^{5} \\
& z_{\mathrm{NP}}=\frac{11-5 \sqrt{5}}{2}=-\left(\frac{\sqrt{5}-1}{2}\right)^{5}
\end{aligned}
$$

It is also useful to discuss the activity function $a(\tau)$ in terms of fundamental domains. If $\mathscr{F}{ }_{5}^{(1)}$ denotes a fundamental domain for $\Gamma_{1}[5]$, then a fundamental domain $\mathscr{F}_{5}$ for $I[5]$ can be chosen to be ${ }^{(14)}$

$$
\widetilde{F}_{5}=\bigcup_{r=0}^{4}\left(\mathscr{F}_{5}^{(1)}\right)_{U^{r}}
$$

[this follows from the coset decomposition (B.3)], where $\left(\mathscr{F}_{5}^{(1)}\right)_{A}$ denotes the image of $\mathscr{F}_{5}^{(1)}$ under $A \in S L(2, \mathbb{Z})$ and $U=\left(\begin{array}{ll}1 & 1 \\ 0 & 1\end{array}\right)$. Now $\zeta$ is univalent on $\mathscr{F}_{5}$, but, of course, $\zeta^{5}$ is not. Given $z \in \hat{\mathbb{C}}$, there are five points $\tau \in \hat{\mathscr{F}}_{5}$ $\left(=\mathscr{F}_{5} \cup\right.$ cusps $)$ such that $\zeta^{5}(\tau)=z$. Since

$$
\zeta(\tau+1)=\omega_{5} \zeta(\tau), \quad \omega_{5}=\exp (2 \pi i / 5)
$$

these five points are related via $\tau \rightarrow \tau+1$ and are, in view of (3.7), equivalent points for $\Gamma_{1}[5]$; hence, $\zeta^{5}$ is univalent on $\mathscr{F}\left(\frac{11}{5}\right.$.

Explicitly, $\mathscr{F}_{5}^{(1)}$ may be chosen to be ${ }^{(14)}$

$$
\mathscr{F}_{5}^{(1)}=\bigcup_{i=1}^{4}\left(\bigcup_{r=0}^{N_{i}-1} \mathscr{F}_{U^{r}}\right)_{A_{i}^{-1}}
$$

where an inequivalent set of cusps is $\{i \infty, 0,2 / 5,1 / 2\}, N_{i}$ is the width of cusp $i, \mathscr{F}$ is the standard fundamental domain for $S L(2, \mathbb{Z})$, and

$$
A_{1}=\left(\begin{array}{ll}
1 & 0 \\
0 & 1
\end{array}\right), \quad A_{2}=\left(\begin{array}{rr}
0 & -1 \\
1 & 0
\end{array}\right), \quad A_{3}=\left(\begin{array}{ll}
2 & -1 \\
5 & -2
\end{array}\right), \quad A_{4}=\left(\begin{array}{ll}
1 & -1 \\
2 & -1
\end{array}\right)
$$

(see Appendix B). To connect with the previous discussion of $z(\tau)$, observe that at these cusps (see Table III) $z \in\left\{0, \infty, z_{c}, z_{\mathrm{NP}}\right\}$. 


\section{4. $\mathrm{K}=\mathrm{K}(z)$ AND $\rho=\rho(z)$}

For $\tau \in \mathscr{H}, J^{\prime}(\tau) \neq 0$ except at $\tau=i, \tau=\rho\left(=e^{2 \pi i / 3}\right)$, and the points $S L(2, \mathbb{Z})$-equivalent to $i$ and $\rho$. Recalling that $J(i)=1$ and $J(\rho)=0$, we have (picking a branch) that $\tau(J)$ is holomorphic in the cut $J$ plane with branch points at $0,1, \infty$. Noting that $\kappa$ and $\rho$ are holomorphic in $\tau$, it follows from (3.5) that (again picking a branch) $\kappa=\kappa(z)$ and $\rho=\rho(z)$ are holomorphic in the cut $z$ plane. The possible branch points are $0, \infty, z_{c}$, $z_{\mathrm{NP}}$, and those $z$ for which $J=1$ and $J=0$. Thus, this quick analysis tells us which points to focus on. We now present a more detailed analysis.

\subsection{Disordered Regime}

In the disordered phase, $\kappa(\tau)$ and $\rho(\tau)$ are modular functions on $\Gamma_{1}[30]$. The group $\Gamma_{1}[30]$ is a subgroup of index 24 in $\Gamma_{1}[5]$ (see Appendix B); and so a fundamental domain for $\Gamma_{1}$ [30] can be made from 24 fundamental domains, appropriately patched together, of $\Gamma_{1}[5]$. This means that when we choose $z$ as the independent variable, $\kappa=\kappa(z)$ and $\rho=\rho(z)$ will be a 24-sheeted covering of the $z$ plane. One of these sheets will be the physical sheet.

We now show that the points arising from $J=0,1$ are not branch points. In the modular group, $\tau=i, \rho$ are elliptic fixed points; but in the congruence subgroups $\Gamma[N]$ these are not fixed points, ${ }^{(11)}$ and the local variables are simply $(\tau-i)$ and $(\tau-\rho)$, respectively. From this and the fact that $\zeta(\tau)$ is univalent on $\mathscr{F}_{5}$ we conclude that at $\tau^{\prime}[=S L(2, \mathbb{Z})$-equivalent point of $\rho$ or $i]$ we have

$$
z-z^{\prime}=\alpha\left(\tau-\tau^{\prime}\right)+\cdots, \quad \alpha \neq 0
$$

and so the series may be inverted to give $\tau=\tau(z)$ holomorphic in some neighborhood of $z^{\prime}$. Hence $\kappa=\kappa(\tau(z))$ and $\rho=\rho(\tau(z))$ are also holomorphic. Thus, we have only to examine the points $0, \infty, z_{c}, z_{\mathrm{NP}}$.

Four inequivalent cusps of $\Gamma_{1}[5]$ can be chosen to be $i \infty, 0,2 / 5,1 / 2$ (see Appendix B). At each cusp we introduce a local uniformizing variable (Table I) and use the transformation properties of $\eta(\tau)$ and $\eta_{\mathrm{g}}(\tau ; N)$ to write a local expansion for $s$. These local expansions are given in Table III. To analyze $\kappa=\kappa(z)$ and $\rho=\rho(z)$ we first find a set of $\Gamma_{1}[30]$ inequivalent cusps and group them into subsets of $\Gamma_{1}[5]$-equivalent cusps. This is done in Table II, Appendix B. There are 32 inequivalent cusps and hence 32 local expressions for $\kappa=\kappa(\tau)$ and for $\rho=\rho(\tau)$ (see Table IV and V). Most of these represent $\kappa$ and $\rho$ on unphysical sheets.

To determine the behavior in $\%$, we now eliminate the local uniformizing variable $x$ (Appendix B defines " $x$ "!). To determine $\kappa$ on the physical 
sheet, we must restrict $\tau$ to lie in the fundamental domain $\mathscr{F}_{5}^{(1)}$ as defined by (3.9) and not one of its images used to construct the fundamental domain $\mathscr{F}_{30}^{(1)}$ of $\Gamma_{1}[30]$. This is the case since an elimination of the local variable $x$ in the expansion at $i \infty 0$ of $\kappa=\kappa(\tau)$ and $z=z(\tau)$ gives the convergent physical small-activity expansion of $\kappa=\kappa(z)$. If one of the $\Gamma_{1}[5]$ equivalent but $\Gamma_{1}[30]$-inequivalent cusps to $i \infty$ is chosen, then the smallactivity expansion of $\kappa$ on an unphysical sheet results. At $z=0$ on the unphysical sheets, $\kappa(z)$ has sixth-root, cube-root, and square-root singularities and one additional holomorphic point.

The behavior of $\kappa=\kappa(z)$ near $z=z_{c}$, on the physical sheet has already been discussed by Baxter. ${ }^{(1-3)}$ Defining

$$
t=\left|1-z / z_{c}\right|
$$

one inverts the local expansion $z=z(\tau)$ at $1 / 2$ in Table III to obtain

$$
|x|=t^{1 / 3} F(t), \quad x=\text { local } \Gamma_{1}[30] \text {-variable at } 1 / 2, \quad z<z_{c}
$$

where $F(t)$ is a convergent power series in $t, F(0) \neq 0$. Note (i) take $x \rightarrow x^{3}$ in Table III to get a local $\Gamma_{1}[30]$ variable (see discussion in Appendix B) and (ii) $x<0$ for $t$ real. From Table IV it follows that the local expansion of $\kappa=\kappa(\tau)$ at the cusp $1 / 2$ will be in powers of the variables $x^{3}$ and $x^{5}$. Thus, in terms of $t, \kappa$ will have the convergent Puiseux expansion

$$
\kappa(z)=\sum_{n_{1}, n_{2} \geqslant 0} \kappa_{n_{1}, n_{2}} t^{n_{1}+(5 / 3) n_{2}}, \quad z \rightarrow z_{c}^{-}
$$

Explicitly, the first few terms are

$$
\begin{aligned}
\frac{\kappa}{\kappa_{c}}=1 & -\frac{1}{2}\left(1-\frac{1}{\sqrt{5}}\right) t+\frac{3}{25 \sqrt{5}} t^{5 / 3}-\frac{3}{25} t^{2} \\
& +\frac{1}{125}(2+\sqrt{5}) t^{8 / 3}+\frac{1}{25}\left(\frac{7}{25} \sqrt{5}-\frac{3}{2}\right) t^{3}+O\left(t^{10 / 3}\right)
\end{aligned}
$$

where

$$
\kappa_{c}=\frac{3 \sqrt{3}}{10} \frac{\sin ^{2}(2 \pi / 5)}{\sin ^{3}(\pi / 5)}=\left[\frac{27}{250}(25+11 \sqrt{5})\right]^{1 / 2}
$$

On the unphysical sheets the behavior of $k(z)$ near $z_{c}$ can be quite different, as can be seen from Table IV. For example, at the cusp 1/12, $\kappa(\%)$ has a pole of order 5 , the leading term in the Laurent expansion being given by

$$
\kappa(z) \sim i \frac{\sin ^{2}(2 \pi / 5)}{\sin ^{3}(\pi / 5)}\left(\frac{z}{z_{c}}-1\right)^{-5}
$$


On other sheets there are square-root and sixth-root branch points at $z=z_{c}$. We know of no statistical mechanical interpretation of these singularities on the unphysical sheets.

The other singularity of $\kappa(z)$ in the finite $z$ plane occurs ar $z=z_{\mathrm{NP}}$. A similar analysis as above shows that if $s=\left|1-z / z_{\mathrm{NP}}\right|$, then

$$
x=s^{1 / 6} F(s), \quad x=\text { local } \Gamma_{1}[30] \text { variable at cusp } 0
$$

where $F(s)$ is a convergent power series in $s, F(0) \neq 0$ [not the same $F$ as in (4.2)]. From Table IV we conclude that at the unphysical singularity ${ }_{{ }_{\mathrm{NP}}}$ on the physical sheet

$$
\kappa(z)=\sum_{n_{1}, n_{2} \geqslant 0} \kappa_{n_{1}, n_{2}}^{(\mathrm{P})} s^{n_{1}+(5 / 6) n_{2}}, \quad z \rightarrow z_{\mathrm{NP}}^{+}
$$

The coefficients $\kappa_{n_{1}, n_{2}}^{(\mathrm{NP})}$ can be computed explicitly from the tables if so desired. It is this singularity that determines the radius of convergence of the physical small- $z$ cluster expansion. On the other sheets the behavior at $z_{\mathrm{NP}}$ can be either a square-root branch point, cube-root branch point, or a pole.

The cusp $2 / 5$ corresponds to $z=\infty$ on the physical sheet. Here $\kappa$ has a sixth-root branch point with a square-root and cube-root branch points on unphysical sheets. On two unphysical sheets $\kappa$ has a pole (look at cusps $7 / 30$ and $13 / 30$ ).

In Table $V$ the local expansions for the density in the disordered regime are given. Proceeding as above, we see that, at $z=z_{c}, \rho(z)$ has on the physical sheet a convergent expansion of the form

$$
\rho(z)=\sum_{n_{1}, n_{2}, n_{3} \geqslant 0} \rho_{n_{1}, n_{2}, n_{3}} t^{(2 / 3) n_{1}+n_{2}+(5 / 3) n_{3}}
$$

Explicitly,

$$
\begin{aligned}
\sqrt{5}\left(\rho_{c}-\rho\right)= & \frac{1}{5} t^{2 / 3}-\frac{1}{5 \sqrt{5}} t+\frac{1}{15}\left(1+\frac{1}{5 \sqrt{5}}\right) t^{5 / 3} \\
& -\frac{1}{10}\left(\frac{1}{25}+\frac{1}{\sqrt{5}}\right) t^{2}+\frac{1}{125 \sqrt{5}} t^{7 / 3}+O\left(t^{8 / 3}\right), \quad z \rightarrow z_{c}^{-}
\end{aligned}
$$

where $\rho_{c}=(5-\sqrt{5}) / 10$. At $z=z_{N P} \phi \rho$ has on the physical sheet the convergent expansion

$\rho(z)=-\rho_{0} s^{-1 / 3} \sum \rho_{n_{1}, n_{2}, n_{3}} s^{(1 / 3) n_{1}+2 n_{2}+(5 / 3)\left(2 n_{3}+1\right)}, \quad z \rightarrow z_{\mathrm{NP}}^{+}$ 
The valence of a modular function is the number of $\tau$ in a fundamental domain (including the cusps) that solve $f(\tau)=c$ for a fixed $c \in \mathbb{C} \cup\{\infty\}$. Since the valence is independent of $c$, we can choose a convenient value, say $c=\infty$. From (2.1) we see that $\kappa(\tau)$ is holomorphic for $\tau \in \mathscr{H}$ and is only $\infty$ at the cusps. From Table IV we see that $\infty$ is assumed (counting multiplicity) 22 times. Similarly, the valence of $\rho(\tau)$ is 8 .

\subsection{Ordered Regime}

As in the disordered regime, the only possible branch points occur at $0, z_{c}, z_{\mathrm{NP}}$, and $\infty$. Noting that $\left[\Gamma_{1}[5]: \Gamma_{1}[15]\right]=8$ and $\left[\Gamma_{1}[5]: \Gamma_{1}[45]\right]=72$ (see Appendix B), it follows that $\kappa^{3}(z)$ and $R^{3}(z)$ continue analytically to an eight-sheeted covering of the $z$ plane and $\rho_{2}(z)$ to a 72-sheeted covering. In the tables of Appendix B, we present a cusp analysis sufficient only to describe the behavior on the physical sheet.

In the ordered regime, it is in the neighborhood of the cusp at 0 that gives the behavior on the physical sheet of quantities (2.4)-(2.7) near $z_{c}$. Introducing $t$ as before [see (4.1)], one inverts local expansions to find

$$
\kappa(z)=\sum_{n_{1}, n_{2} \geqslant 0} \kappa_{n_{1} n_{2}} t^{n_{1}+(5 / 3) m_{2}}, \quad t \rightarrow 0
$$

Explicitly, the first few terms are

$$
\begin{aligned}
\frac{\kappa}{\kappa_{c}}=1 & +\frac{1}{2}\left(1-\frac{1}{\sqrt{5}}\right) t+\frac{3}{25 \sqrt{5}} t^{5 / 3}-\frac{1}{25}(6-\sqrt{5}) t^{2} \\
& +\frac{1}{125}(4 \sqrt{5}-1) t^{8 / 3}+\left(\frac{39}{250}-\frac{29}{1250} \sqrt{5}\right) t^{3}+O\left(t^{10 / 3}\right)
\end{aligned}
$$

with $z \rightarrow z_{c}^{+}$.

Similarly,

$$
R(z)=t^{1 / 9} \sum_{n_{1}, n_{2} \geq 0} R_{n_{1} n_{2}} t^{n_{1}+(5 / 3) n_{2}}
$$

and

$$
\rho_{2}(z)=\rho_{c} \sum_{n_{1}, n_{2} \geqslant 0} \rho_{n_{1} n_{2}}^{(2)} t^{n_{1}+(1 / 9) n_{2}}
$$

for $t \rightarrow 0$ on the physical sheet. The cusp $2 / 5$ gives the $z$ near zero properties for the physical sheet. From Table VIII we see that $\kappa$ has a cube-root singularity. 


\section{APPENDIX A. DEDEKIND FUNCTIONS}

For $\tau \in \mathscr{H}$ the Dedekind eta function $\eta(\tau)$ is defined by ${ }^{(11)}$

$$
\eta(\tau)=e^{\pi i \tau / 12} \prod_{m=1}^{\infty}\left(1-e^{2 \pi i m \tau}\right)
$$

For $\mathbf{g}=(g, h) \in \mathbb{Z}_{N} \times \mathbb{Z}_{N}$ we have the generalized Dedekind functions ${ }^{(11)}$

$$
\begin{gathered}
\eta_{\mathbf{g}}(\tau ; N)=\alpha(\mathbf{g}) e^{\pi i P_{2}(g / N) \tau} \prod_{\substack{m>0 \\
m \equiv g(\bmod N)}}\left(1-\omega_{N}^{h} e^{(2 \pi i / N) \tau m}\right) \\
\times \prod_{\substack{m>0 \\
m \equiv-g(\bmod N)}}\left(1-\omega_{N}^{-h} e^{2 \pi i \tau m / N}\right)
\end{gathered}
$$

with

$$
\begin{gathered}
\alpha(\mathbf{g})= \begin{cases}\left(1-\omega_{N}^{-h}\right) e^{\pi i P_{1}(h / N)} & \text { if } g \equiv 0 \text { and } h \neq 0 \bmod N \\
1 & \text { otherwise }\end{cases} \\
\omega_{N}=\exp (2 \pi i / N) \\
P_{1}(x)=x-[x]-1 / 2 \\
P_{2}(x)=(x-[x])^{2}-(x-[x])+1 / 6
\end{gathered}
$$

For $A=\left(\begin{array}{cc}a & b \\ c & d\end{array}\right) \in S L(2, \mathbb{Z}), \eta(\tau)$ and $\eta_{\mathrm{g}}(\tau ; N)$ transform as follows:

$$
\eta\left(\frac{a \tau+b}{c \tau+d}\right)=\exp \left(\frac{\pi i}{12} \phi(A)\right)\left(\frac{c \tau+d}{i}\right)^{1 / 2} \eta(\tau)
$$

and for $\mathbf{g} \equiv(0,0)(\bmod N)$,

$$
\eta_{\mathrm{g}}\left(\frac{a \tau+b}{c \tau+d} ; N\right)=\exp \left[\pi_{\mathrm{g}}(A)\right] \eta_{\mathrm{g}^{\prime}}(\tau ; N)
$$

where $\mathbf{g}^{\prime}=\left(g^{\prime}, h^{\prime}\right)=(g, h) A$,

$$
\phi(A)= \begin{cases}\frac{a+d}{c}-12 \operatorname{sgn}(c) S(d,|c|) & \text { for } c \neq 0 \\ \frac{b}{d} & \text { for } c=0\end{cases}
$$




$$
\pi_{\mathbf{g}}(A)= \begin{cases}\pi i\left[\frac{a}{c} P_{2}\left(\frac{g^{\prime}}{n}\right)+\frac{d}{c} P_{2}\left(\frac{g}{N}\right)-2 \operatorname{sgn}(c) S_{\mathrm{g}}^{N}(a, c)\right] & \text { for } c \neq 0 \\ \pi i \frac{b}{d} P_{2}\left(\frac{g}{n}\right) & \text { if } c=0\end{cases}
$$

and $S(a, c)$ is the Dedekind sum

$$
S(a, c)=\sum_{v \bmod c}\left(\left(\frac{v}{c}\right)\right)\left(\left(\frac{a v}{c}\right)\right)
$$

and $S_{g, h}^{N}(a, c)$ is the generalized Dedekind sum

$$
S_{g, h}^{N}(a, c)=\sum_{v \bmod c}\left(\left(\frac{g+v N}{c N}\right)\right)\left(\left(\frac{g^{\prime}+a v N}{c N}\right)\right)
$$

where $((x))=x-[x]-\frac{1}{2}+\frac{1}{2} \delta(x)$ with $\delta(x)=1$ if $x \in \mathbb{Z}$ and 0 otherwise.

In particular, for $A \in \Gamma[N]$ and $\mathbf{g} \equiv(0,0)(\bmod N)$,

$$
\eta_{\mathrm{g}}(A \tau ; N)=\exp \left[\pi_{\mathrm{g}}(A)\right] \eta_{\mathrm{g}}(\tau ; N)
$$

Since $\pi_{\mathbf{g}}(A) \in \mathbb{Q}$, there exists an integer $N_{1}$ such that $\left(\eta_{\mathbf{g}}(\tau ; N)\right)^{N_{1}}$ is a modular function of level $N, \mathbf{g} \equiv(0,0)$. According to Schoeneberg, ${ }^{(11)}$ the choice $N_{1}=12 N /(6, N)$ works. The periods $\pi_{\mathrm{g}}(A)$ satisfy ${ }^{(11)}$

$$
\pi_{\mathbf{g}}(A B)=\pi_{\mathbf{g}}(A)+\pi_{t_{A g}}(B)
$$

so that if $A \in \Gamma[N]$, we have

$$
\pi_{\mathbf{g}}(A B)=\pi_{\mathbf{g}}(A)+\pi_{\mathrm{g}}(B)
$$

\section{APPENDIX B. CONGRUENCE SUBGROUPS AND LOCAL EXPANSIONS}

We define the standard congruence subgroups

$$
\begin{gathered}
\Gamma[N]=\left\{A \in S L(2, \mathbb{Z}): A \equiv \pm\left(\begin{array}{ll}
1 & 0 \\
0 & 1
\end{array}\right) \bmod N\right\} \\
\Gamma_{1}[N]=\left\{A \in S L(2, \mathbb{Z}): A \equiv \pm\left(\begin{array}{ll}
1 & * \\
0 & 1
\end{array}\right) \bmod N\right\}
\end{gathered}
$$

and take $\Gamma=\Gamma[1]$. 
If $U=\left(\begin{array}{ll}1 & 1 \\ 0 & 1\end{array}\right)$, then we have the coset decomposition

$$
\Gamma_{1}[N]=\Gamma[N] \cup U \Gamma[N] \cup \cdots \cup U^{N-1} \Gamma[N]
$$

Thus, $\left[\Gamma_{1}[N]: \Gamma[N]\right]=N$. Using ${ }^{(11)}$

$$
[\Gamma: \Gamma[N]]=\frac{1}{2} N^{3} \prod_{p \mid N}\left(1-\frac{1}{p^{2}}\right)
$$

we conclude that

$$
\left[\Gamma: \Gamma_{1}[N]\right]=\frac{1}{2} N^{2} \prod_{p \mid N}\left(1-\frac{1}{p^{2}}\right)
$$

In particular, $\left[\Gamma: \Gamma_{1}[5]\right]=12$ and $\left[\Gamma: \Gamma_{1}[30]\right]=288$, so that

$$
\left[\Gamma_{1}[5]: \Gamma_{1}[30]\right]=24
$$

We write a cusp $x / y \in \mathbb{Q}$ as $\left(\begin{array}{l}x \\ y\end{array}\right)$. The it is known ${ }^{(11,15)}$ that a set of inequivalent cusps of $\Gamma[N]$ is $\pm\left(\begin{array}{l}x \\ y\end{array}\right),(x, y)=1, x, y \in \mathbb{Z}_{N}$ with $-\left(\begin{array}{l}x \\ y\end{array}\right)$ identified with $\left(\begin{array}{l}x \\ y\end{array}\right)$. In terms of these cusps the cusps of $\Gamma_{1}[N]$ can be ${ }^{(15)}$ identified with the orbits $\left\{ \pm\left({ }^{x+b y}\right): b \in \mathbb{Z}_{N}\right\}$. In particular, $\Gamma_{1}[5]$ has four inequivalent cusps, which can be chosen as $\{i \infty, 0,2 / 5,1 / 2\}$, and $\Gamma_{1}$ [30] has 32 inequivalent cusps (see Table II).

In the neighborhood of a cusp $h / k,(h, k)=1$, we write

$$
\tau=h / k+i t / k, \quad \operatorname{Re} t>0
$$

Then if $h^{\prime}$ solves the congruence

$$
h h^{\prime} \equiv-1(\bmod k)
$$

Table I. Cusp Data for $\Gamma_{1}[5], \omega_{N}=\exp (2 \pi i / N)$

\begin{tabular}{ccc}
$\begin{array}{c}\text { Cusp } \\
h\end{array}$ & Cusp width & \multicolumn{1}{c}{ Local uniformizing variable } \\
$\frac{h}{k}$ & $\kappa_{5}$ & $x=\exp \left[\frac{2 \pi i}{\kappa_{5}}\left(\frac{h^{\prime}}{k}+\frac{i}{k t}\right)\right], e(t)=\exp \left(-\frac{2 \pi}{t}\right)$ \\
\hline$i \infty$ & 1 & $e(t)$ \\
0 & 5 & $e(5 t)$ \\
$2 / 5$ & 1 & $\omega_{5}^{2} e(5 t)$ \\
$1 / 2$ & 5 & $-e(10 t)$ \\
\hline
\end{tabular}


Table II. Cusp Data for $\Gamma_{1}[30], \omega_{N}=\exp (2 \pi i / N)$

\begin{tabular}{|c|c|c|}
\hline Cusp & $\begin{array}{l}\text { Cusp width } \\
\kappa_{30}\end{array}$ & $\begin{array}{c}\text { Local uniformizing } \\
\text { variable } x, e(t)=\exp (-2 \pi / t)\end{array}$ \\
\hline$i \infty$ & 1 & $e(t)$ \\
\hline $11 / 30$ & 1 & $\omega_{30}^{19} e(30 t)$ \\
\hline $4 / 5$ & 6 & $\omega_{5} e(30 t)$ \\
\hline $1 / 5$ & 6 & $\omega_{5}^{4} e(30 t)$ \\
\hline $1 / 10$ & 3 & $\omega_{10}^{3} e(30 t)$ \\
\hline $9 / 10$ & 3 & $\omega_{10}^{7} e(30 t)$ \\
\hline $1 / 15$ & 2 & $\omega_{15}^{7} e(30 t)$ \\
\hline $4 / 15$ & 2 & $\omega_{15}^{13} e(30 t)$ \\
\hline $2 / 5$ & 6 & $\omega_{5}^{2} e(30 t)$ \\
\hline $3 / 5$ & 6 & $\omega_{5}^{3} e(30 t)$ \\
\hline $3 / 10$ & 3 & $\omega_{10} e(30 t)$ \\
\hline $7 / 10$ & 3 & $\omega_{10}^{9} e(30 t)$ \\
\hline $2 / 15$ & 2 & $\omega_{15}^{11} e(30 t)$ \\
\hline $7 / 15$ & 2 & $\omega_{15} e(30 t)$ \\
\hline $7 / 30$ & 1 & $\omega_{30}^{17} e(30 t)$ \\
\hline $13 / 30$ & 1 & $\omega_{30}^{23} e(30 t)$ \\
\hline 0 & 30 & $e(30 t)$ \\
\hline $1 / 11$ & 30 & $\omega_{11}^{4} e(330 t)$ \\
\hline $1 / 4$ & 15 & $\omega_{4} e(60 t)$ \\
\hline $1 / 14$ & 15 & $\omega_{14}^{13} e(210 t)$ \\
\hline $1 / 9$ & 10 & $\omega_{9}^{8} e(90 t)$ \\
\hline $2 / 9$ & 10 & $\omega_{9}^{4} e(90 t)$ \\
\hline $1 / 6$ & 5 & $\omega_{6} e(30 t)$ \\
\hline $5 / 6$ & 5 & $\omega_{6}^{5} e(30 t)$ \\
\hline $1 / 2$ & 15 & $-e(30 t)$ \\
\hline $1 / 8$ & 15 & $\omega_{8} e(120 t)$ \\
\hline $1 / 7$ & 30 & $\omega_{7}^{3} e(210 t)$ \\
\hline $1 / 13$ & 30 & $\omega_{13}^{3} e(390 t)$ \\
\hline $1 / 3$ & 10 & $\omega_{3}^{2} e(30 t)$ \\
\hline $2 / 3$ & 10 & $\omega_{3} e(30 t)$ \\
\hline $1 / 12$ & 5 & $\omega_{12}^{7} e(60 t)$ \\
\hline $5 / 12$ & 5 & $\omega_{12}^{11} e(60 t)$ \\
\hline
\end{tabular}


the matrix

$$
A=\left(\begin{array}{cc}
h & -\left(h h^{\prime}+1\right) / k \\
k & -h^{\prime}
\end{array}\right)
$$

is in $S L(2, \mathbb{Z})$ and

$$
A \tau^{\prime}=\tau
$$

with

$$
\tau^{\prime}=h^{\prime} / k+i / k t
$$

The width of the cusp $h / k$ of $\Gamma_{1}[N]$ is defined to be the smallest integer $\kappa$ such that $A U^{\kappa} A^{-1} \in \Gamma_{1}[N]$. A local uniformizing variable $x$ of the cusp $h / k$ is

$$
x=e^{2 \pi i \tau^{\prime} / \kappa}
$$

Observe that since $h^{\prime}$ is determined only $\bmod k, x$ is determined up to a $\kappa$ th root of unity. This ambiguity is just a local change of coordinates and we will use this freedom to choose a local variable $x$ that gives the "nicest" results.

In Table I a set of inequivalent cusps, cusp widths, and local uniformizing variables for $\Gamma_{1}$ [5] are given. In Table II the same data are given for $\Gamma_{1}[30]$. In Table III the local expansions of $\zeta^{5}(\tau)$ at each of the inequivalent cusps of Table I are presented. Note that the " $x$ " is the local variable as defined in Table I. In Table IV the local expansions of $\kappa(\tau)$, disordered regime, at each of the inequivalent cusps of Table II are presented. Again, note that the " $x$ " is the local variable appearing in Table II.

Table III. Local Expansion of $\zeta^{5}(\tau)$ at Inequivalent Cusps of $\Gamma_{1}[5]^{a}$

\begin{tabular}{cl}
\hline Cusp & Local expansion of $\zeta^{5}(\tau)$ \\
\hline$i \infty$ & $x([5,1] /[5,2])^{5}$ \\
0 & $\zeta_{1}^{5}\left(\left[1 ; \omega_{5}\right] /\left[1 ; \omega_{5}^{2}\right]\right)^{5}$ \\
$2 / 5$ & $-x^{-1}([5,2] /[5,1])^{5}$ \\
$1 / 2$ & $-\zeta_{2}^{5}\left(\left[1 ; \omega_{5}^{2}\right] /\left[1 ; \omega_{5}\right]\right)^{5}$ \\
\hline$a \zeta_{1}=(\sqrt{5}-1) / 2, \zeta_{2}=(\sqrt{5}+1) / 2$, & $\omega_{5}=\exp (2 \pi i / 5)$.
\end{tabular}


Table IV. Local Expansions of $k(T)$, Disordered Regime, at Inequivalent Cusps of $\Gamma_{1}[30]$ As Given in Table II ${ }^{a}$

\begin{tabular}{|c|c|}
\hline Cusp & Local expansion \\
\hline $\begin{array}{l}i \infty \\
11 / 30\end{array}$ & $\pm[5]^{2}[5,1]^{2}[6,3][6,2] /[5,2]^{3}[6,1][6]^{2}$ \\
\hline $\begin{array}{l}4 / 5 \\
1 / 5\end{array}$ & $\pm 12 i \sqrt{3} x^{6}[30]^{2}[30,6]^{2}[1 ;-1]\left[1 ; \omega_{3}\right] /[30,12]^{3}\left[1 ; \omega_{6}\right][1]^{2}$ \\
\hline $\begin{array}{l}1 / 10 \\
9 / 10\end{array}$ & $\pm 3 i \sqrt{3} x^{3}[15]^{2}[15,3]^{2}[2,1]\left[2 ; \omega_{2}\right] /[15,6]^{3}\left[2,1 ; \omega_{3}\right][2]^{2}$ \\
\hline $\begin{array}{l}1 / 15 \\
4 / 15\end{array}$ & $\pm 4 x^{2}[10]^{2}[10,2]^{2}[3 ;-1][3,1] /[10,4]^{3}[3,1 ;-1][3]^{2}$ \\
\hline $\begin{array}{l}3 / 5 \\
2 / 5\end{array}$ & $\pm 12(i \sqrt{3})[30]^{2}[30,12]^{2}[1 ;-1]\left[1 ; \omega_{3}\right] /[30,6]^{3}\left[1 ; \omega_{6}\right][1]^{2}$ \\
\hline $\begin{array}{l}7 / 10 \\
3 / 10\end{array}$ & $\pm 3(i \sqrt{3})[15]^{2}[15,6]^{2}[2,1]\left[2 ; \omega_{3}\right] /[15,3]^{3}\left[2,1 ; \omega_{3}\right][2]^{2}$ \\
\hline $\begin{array}{l}7 / 15 \\
2 / 15\end{array}$ & $\pm 4[10]^{2}[10,4]^{2}[3 ;-1][3,1] /[10,2]^{3}[3,1 ;-1][3]^{2}$ \\
\hline $\begin{array}{l}7 / 30 \\
13 / 30\end{array}$ & $\pm x^{-1}[5]^{2}[5,2]^{2}[6,3][6,2] /[5,1]^{3}[6,1][6]^{2}$ \\
\hline $\begin{array}{l}0 \\
1 / 11\end{array}$ & $\pm(6 \sqrt{3} / 5) c_{1}[6]^{2}\left[6 ; \omega_{5}\right]^{2}[5 ;-1]\left[5 ; \omega_{3}\right] /\left[6 ; \omega_{5}^{2}\right]^{3}\left[5 ; \omega_{6}\right][5]^{2}$ \\
\hline $\begin{array}{l}1 / 14 \\
1 / 4\end{array}$ & $\pm(3 \sqrt{3} / 10) c_{1}[3]^{2}\left[3 ; \omega_{5}\right]^{2}[10,5]\left[10 ; \omega_{3}\right] /\left[3 ; \omega_{5}^{2}\right]^{3}\left[10,5 ; \omega_{3}\right][10]^{2}$ \\
\hline $\begin{array}{l}1 / 9 \\
2 / 9\end{array}$ & $\pm\left(2 i c_{1} / 5\right)[2]^{2}\left[2 ; \omega_{5}\right]^{2}[15 ;-1][15,5] /\left[2 ; \omega_{5}^{2}\right]^{3}[15,5 ;-1][15]^{2}$ \\
\hline $\begin{array}{l}5 / 6 \\
1 / 6\end{array}$ & $\pm(i / 10) c_{1} x^{-5}[1]^{2}\left[1 ; \omega_{5}\right]^{2}[30,15][30,10] /\left[1 ; \omega_{5}^{2}\right]^{3}[30,5][30]^{2}$ \\
\hline $\begin{array}{l}1 / 2 \\
1 / 8\end{array}$ & $\pm(3 \sqrt{3} / 10) c_{2}[3]^{2}\left[3 ; \omega_{5}^{2}\right]^{2}[10,5]\left[10 ; \omega_{3}\right] /\left[3 ; \omega_{5}\right]^{3}\left[10,5 ; \omega_{3}\right][10]^{2}$ \\
\hline $\begin{array}{l}1 / 13 \\
1 / 7\end{array}$ & $\pm(6 \sqrt{3} / 5) c_{2}[6]^{2}\left[6 ; \omega_{5}^{2}\right]^{2}[5 ;-1]\left[5 ; \omega_{3}\right] /\left[6 ; \omega_{5}\right]^{3}\left[5 ; \omega_{6}\right][5]^{2}$ \\
\hline $\begin{array}{l}2 / 3 \\
1 / 3\end{array}$ & $\pm\left(2 i c_{2} / 5\right)[2]^{2}\left[2 ; \omega_{5}^{2}\right][15 ;-1][15,5] /\left[2 ; \omega_{5}\right]^{3}[15,5 ;-1][15]^{2}$ \\
\hline $\begin{array}{l}1 / 12 \\
5 / 12\end{array}$ & $\pm(i / 10) c_{2} x^{-5}[1]^{2}\left[1 ; \omega_{5}^{2}\right]^{2}[30,15][30,10] /\left[1 ; \omega_{5}\right]^{3}[30,5][30]^{2}$ \\
\hline
\end{tabular}

${ }^{a}$ The lower sign refers to the second cusp. The local variable " $x$ " is defined by Table II, $c_{1}=$ $\sin ^{2}(\pi / 5) / \sin ^{3}(2 \pi / 5), c_{2}=\sin ^{2}(2 \pi / 5) / \sin ^{3}(\pi / 5), \omega_{N}=\exp (2 \pi i / N)$. 
In general, if $x^{\prime}$ is the local variable for $h / k+i t / k$ in $\Gamma_{1}[5]$ and $x^{\prime \prime}$ is the local variable about the same cusp but now viewed in $\Gamma_{1}[30]$, then, using (B.9) and (B.10), we have

$$
x^{\prime}=\left(x^{\prime \prime}\right)^{\kappa_{30}^{\prime}}
$$

where

$$
\kappa_{30}=\kappa_{5} \kappa_{30}^{\prime}
$$

Thus, in words, if one has the local expansion of a $\Gamma_{1}[5]$-invariant function at $h / k+i t / k$, the corresponding local expansion in $\Gamma_{1}[30]$ is obtained by replacing $x^{\prime}$ in the $\Gamma_{1}[5]$ expansion by $\left(x^{\prime \prime}\right)^{\kappa_{30}}$. Finally, we have, of course, that the local expansion of a $\Gamma_{1}[5]$-invariant function at equivalent cusps is just the same expansion with the interpretation that the " $x$ " is the corresponding local variable.

Table V. Local Expansions of $\rho(T)$, Disordered Regime,

\begin{tabular}{|c|c|}
\hline Cusp & Local expansion \\
\hline $\begin{array}{l}i \infty, 11 / 30 \\
3 / 5,2 / 5\end{array}$ & $-x\{6,3\} /\{2,1\}[5,1][30,12]$ \\
\hline $\begin{array}{l}1 / 5,4 / 5 \\
7 / 30,13 / 30\end{array}$ & $\{6,3\} /\{2,1\}[30,6][5,2]$ \\
\hline $\begin{array}{l}1 / 10,9 / 10 \\
1 / 15,4 / 15\end{array}$ & $\{2,1\} /\{6,3\}[10,2][15,3]$ \\
\hline $\begin{array}{l}0,1 / 11 \\
1 / 12,5 / 12\end{array}$ & $-\rho_{0} x^{-1}\{30,15\} /\{10,5\}\left[6 ; \omega_{5}\right]\left[1 ; \omega_{5}^{2}\right]$ \\
\hline $\begin{array}{l}1 / 2,1 / 8 \\
1 / 3,2 / 3\end{array}$ & $\rho_{c}\{10,5\} /\{30,15\}\left[3 ; \omega_{5}^{2}\right]\left[2 ; \omega_{5}^{2}\right]$ \\
\hline $\begin{array}{l}1 / 4,1 / 14 \\
1 / 9,2 / 9\end{array}$ & $\rho_{\mathrm{NP}}\{10,5\} /\{30,15\}\left[3 ; \omega_{5}\right]\left[2 ; \omega_{5}\right]$ \\
\hline $\begin{array}{l}1 / 7,1 / 13 \\
1 / 6,5 / 6\end{array}$ & $\rho_{0} x^{-1}\{30,15\} /\{10,5\}\left[6 ; \omega_{5}^{2}\right]\left[1 ; \omega_{5}\right]$ \\
\hline $\begin{array}{l}3 / 10,7 / 10 \\
2 / 15,7 / 15\end{array}$ & $x\{2,1\} /\{6,3\}[10,4][15,6]$ \\
\hline
\end{tabular}
at the Inequivalent Cusps of $\Gamma_{1}[30]$ as Given in Table $\mathrm{I}^{a}$

${ }^{a}$ The local variable " $x$ " is defined by Table II, $\rho_{c}=(5-\sqrt{5}) / 10, \rho_{\mathrm{NP}}=(5+\sqrt{5}) / 10$, and $\rho_{0}=$ $[4 \sin (\pi / 5) \sin (2 \pi / 5)]^{-1}$. 
Table VI. Local Variables for a Partial Set of Inequivalent Cusps of $\Gamma_{1}[15]^{a}$

\begin{tabular}{ccc}
\hline Cusp & $\begin{array}{c}\text { Cusp width } \\
\kappa_{\mathrm{1} 5}\end{array}$ & $\begin{array}{c}\text { Local Uniformizing variable } x, \\
e(t)=\exp (-2 \pi / t)\end{array}$ \\
\hline$i \infty$ & 1 & $e(t)$ \\
0 & 15 & $e(15 t)$ \\
$2 / 5$ & 3 & $\omega_{5}^{4} e(15 t)$ \\
$1 / 2$ & 15 & $e(30 t)$ \\
\hline
\end{tabular}

a The cusps chosen are those needed for an analysis on the physical sheet.

To simplify the tables, we use the following notation in Tables III-V and VIII-X:

$$
\begin{aligned}
{[j] } & =\prod_{m=1}^{\infty}\left(1-x^{j m}\right) \\
\{j, k\} & =\prod_{m=1}^{\infty}\left(1-x^{j m-k}\right) \\
{[j, k] } & =\prod_{m=1}^{\infty}\left(1-x^{j m-k}\right)\left(1-x^{j m+k-j}\right) \\
{[j ; a] } & =\prod_{m=1}^{\infty}\left(1-a x^{j m}\right)\left(1-a^{-1} x^{j m}\right) \\
{[j, k ; a] } & =\prod_{m=1}^{\infty}\left(1-a x^{j m-k}\right)\left(1-a^{-1} x^{j m+k-j}\right)
\end{aligned}
$$

Table VII. Local Variables for a Partial Set of Inequivalent Cusps of $r_{1}[45]^{a}$

\begin{tabular}{ccc} 
Cusp & $\begin{array}{c}\text { Cusp width } \\
\kappa_{45}\end{array}$ & $\begin{array}{c}\text { Local uniformizing variable } x, \\
e(t)=\exp (-2 \pi / t)\end{array}$ \\
\hline$i \infty$ & 1 & $e(t)$ \\
0 & 45 & $e(45 t)$ \\
$2 / 5$ & 9 & $\omega_{5}^{3} e(45 t)$ \\
$1 / 2$ & 45 & $-e(90 t)$ \\
\hline
\end{tabular}

${ }^{a}$ The cusps chosen are those needed for an analysis on the physical sheet. 
Table VIII. Local Expansions of $к(\tau)$, Ordered Regime, at the Cusps Given in Table $\mathrm{VJ}^{a}$

\begin{tabular}{cc}
\hline Cusp & Local expansion \\
\hline$i \infty 0$ & $x^{-1 / 3}[5]^{2}[5,2]^{2}[3,1] /[3]^{2}[5,1]^{3}$ \\
0 & $(3 \sqrt{3} / 10) c_{2}[3]^{2}\left[3 ; \omega_{5}^{2}\right]^{2}\left[5 ; \omega_{3}\right] /\left[3 ; \omega_{5}\right]^{3}[5]^{2}$ \\
$2 / 5$ & $(3 \sqrt{3}) e^{-\pi i / 6} x^{3}[15]^{2}[15,3]^{2}\left[1 ; \omega_{3}\right] /[15,6]^{3}[1]^{2}$ \\
$1 / 2$ & $(3 \sqrt{3} / 10) c_{1} e^{-\pi i / 3}[3]^{2}\left[3 ; \omega_{5}\right]^{2}\left[5 ; \omega_{3}\right] /\left[3, \omega_{5}^{2}\right]^{3}[5]^{2}$ \\
\hline
\end{tabular}

${ }^{a}$ The local variable " $x$ " is given in Table VI, $\quad c_{1}=\sin ^{2}(\pi / 5) / \sin ^{3}(2 \pi / 5), \quad c_{2}=$ $\sin ^{2}(2 \pi / 5) / \sin ^{3}(\pi / 5)$, and $\omega_{N}=\exp (2 \pi i / N)$.

Table IX. Local Expansion of $R=\rho_{1}-\rho_{2}$, Ordered Regime, at the Cusps Given in Table VI ${ }^{a}$

\begin{tabular}{cc}
\hline Cusp & Local expansion \\
\hline$i \infty$ & {$[1][5] /[3]^{2}$} \\
0 & $(3 / \sqrt{5}) x^{1 / 3}[15][3] /[5]^{2}$ \\
$2 / 5$ & $\omega_{15} x^{2 / 3}[3][15] /[1]^{2}$ \\
$1 / 2$ & $e^{-\pi i / 3} x^{1 / 3}[3][15] /[5]^{2}$ \\
\hline
\end{tabular}

"The local variable " $x$ " is given in Table VI.

Table X. Local Expansions of $\rho_{2}(\tau)=\rho_{3}(\tau)$. Ordered Regime, at the Cusps Given in Table VIII ${ }^{a}$

\begin{tabular}{cc}
\hline Cusp & Local expansion \\
\hline$i \infty$ & $x^{2}[1][9] /[3]^{2}[5,2][45,18]$ \\
0 & $\rho_{c}[45][5] /[15]^{2}\left[9 ; \omega_{5}^{2}\right]\left[1 ; \omega_{5}^{2}\right]$ \\
$2 / 5$ & {$[45][1] /[3]^{2}[45,9][5,1]$} \\
$1 / 2$ & $\rho_{\mathrm{NP}}[45][5] /[15]^{2}\left[9 ; \omega_{5}\right]\left[1 ; \omega_{5}\right]$ \\
\hline
\end{tabular}

${ }^{a}$ The local variable " $x$ " is given in Table VIII, $\rho_{\mathrm{c}}=(5-\sqrt{5}) / 10, \rho_{\mathrm{NP}}=$ $(5+\sqrt{5}) / 10$. 


\section{APPENDIX C. GENERATORS FOR CONGRUENCE SUBGROUPS}

The group $\Gamma=S L(2, \mathbb{Z})$ is generated by the two matrices

$$
T=\left(\begin{array}{rr}
0 & 1 \\
-1 & 0
\end{array}\right), \quad R=\left(\begin{array}{rr}
0 & 1 \\
-1 & -1
\end{array}\right)
$$

(these are the negatives of the matrices so named in Ref. 11). Let $\mathscr{T}$ be a set of elements of $\Gamma$ consisting of one element from each (right) coset of $\Gamma_{1}[N]$ (that is, a right transversal for $\Gamma_{1}[N]$ in $\Gamma$ ). For each matrix $M$ in $\Gamma$ denote by $\bar{M}$ the element of $\mathscr{T}$ in the coset $\Gamma_{1}[N] M$. A generating set for $\Gamma_{1}[N]$ is $\left\{V T(\overline{V T})^{-1}, V R(\overline{V R})^{-1}: V \in \mathscr{T}\right\}$ (see Robinson, ${ }^{(16)}$ Section 6.1). This set contains at most $2\left[\Gamma: \Gamma_{1}[N]\right]$ elements and, if $\mathscr{T}$ is a Schreier transversal, at most $1+\left[\Gamma: \Gamma_{1}[N]\right]$ nonidentity elements. We used a simple-minded algorithm for constructing a generating set $\mathscr{G}$ for $\Gamma_{1}[N]$. The algorithm builds $\mathscr{G}$ and a Schreier transversal $\mathscr{T}$ concurrently. Initially $\mathscr{G}$ contains just $\left(\begin{array}{rr}-1 & 0 \\ 0 & -1\end{array}\right)$ and $\mathscr{T}$ contains just $\left(\begin{array}{ll}1 & 0 \\ 0 & 1\end{array}\right)$. The algorithm consists of $\left[\Gamma: \Gamma_{1}[N]\right]$ steps, each of which processes an element of $\mathscr{T}$ as follows:

1. Take an unprocessed element $V$ of $\mathscr{T}$.

2. Put $X=V T$.

3. If $\Gamma_{1}[N] X$ has no representative in $\mathscr{T}$, then add $X$ to $\mathscr{T}$ and go to step 5; else put $Y=X(\bar{X})^{-1}$.

4. If $Y, Y^{-1},-Y$, or $-Y^{-1}$ belongs to $\mathscr{G}$, then go to step 5; else add $Y$ to $\mathscr{G}$.

5. Put $X=V R$.

6. If $\Gamma_{1}[N] X$ has no representative in $\mathscr{T}$, then add $X$ to $\mathscr{T}$ and go to step 8; else put $Y=X(\bar{X})^{-1}$.

7. If $Y, Y^{-1},-Y$, or $-Y^{-1}$ belong to $\mathscr{G}$, then go to step 8; else add $Y$ to $\mathscr{G}$.

8. Go to the next step or stop after $\left[\Gamma: \Gamma_{1}[N]\right]$ steps.

The algorithm was implemented in the system CAYLEY (see Cannon ${ }^{(17)}$ ) and run on VAXs at the University of Arizona.

The results are as follows:

$\Gamma_{1}[5]$ is generated by four elements

$$
\left(\begin{array}{rr}
-1 & 0 \\
0 & -1
\end{array}\right)\left(\begin{array}{ll}
1 & 1 \\
0 & 1
\end{array}\right)\left(\begin{array}{rr}
-4 & -1 \\
5 & 1
\end{array}\right)\left(\begin{array}{rr}
11 & 4 \\
-25 & -9
\end{array}\right)
$$


$\Gamma_{1}[15]$ is generated by 18 elements

$$
\begin{aligned}
& \left(\begin{array}{rr}
-1 & 0 \\
0 & -1
\end{array}\right)\left(\begin{array}{ll}
1 & 1 \\
0 & 1
\end{array}\right)\left(\begin{array}{rr}
16 & 5 \\
-45 & -14
\end{array}\right)\left(\begin{array}{rr}
-29 & -12 \\
75 & 31
\end{array}\right)\left(\begin{array}{rr}
-44 & -27 \\
75 & 46
\end{array}\right) \\
& \left(\begin{array}{rr}
31 & 20 \\
-45 & -29
\end{array}\right)\left(\begin{array}{rr}
-14 & -3 \\
75 & 16
\end{array}\right)\left(\begin{array}{rr}
76 & 21 \\
-105 & -29
\end{array}\right)\left(\begin{array}{rr}
-59 & -9 \\
105 & 16
\end{array}\right)\left(\begin{array}{rr}
31 & 17 \\
-135 & -74
\end{array}\right) \\
& \left(\begin{array}{rr}
61 & 16 \\
-225 & -59
\end{array}\right)\left(\begin{array}{rr}
-59 & -7 \\
135 & 16
\end{array}\right)\left(\begin{array}{rr}
-59 & -48 \\
75 & 61
\end{array}\right)\left(\begin{array}{rr}
61 & 47 \\
-135 & -104
\end{array}\right) \\
& \left(\begin{array}{rr}
-149 & -81 \\
195 & 106
\end{array}\right)\left(\begin{array}{rr}
-14 & -1 \\
15 & 1
\end{array}\right)\left(\begin{array}{rr}
31 & 4 \\
-225 & -29
\end{array}\right)\left(\begin{array}{rr}
121 & 64 \\
-225 & -119
\end{array}\right)
\end{aligned}
$$

$\Gamma_{1}[30]$ is generated by 55 elements

$$
\begin{aligned}
& \left(\begin{array}{rr}
-1 & 0 \\
0 & -1
\end{array}\right)\left(\begin{array}{ll}
1 & 1 \\
0 & 1
\end{array}\right)\left(\begin{array}{rr}
61 & 24 \\
-150 & -59
\end{array}\right)\left(\begin{array}{rr}
121 & 34 \\
-210 & -59
\end{array}\right)\left(\begin{array}{rr}
91 & 54 \\
-150 & -89
\end{array}\right) \\
& \left(\begin{array}{rr}
31 & 6 \\
-150 & -29
\end{array}\right) \quad\left(\begin{array}{rr}
-59 & -33 \\
270 & 151
\end{array}\right) \quad\left(\begin{array}{rr}
-89 & -56 \\
240 & 151
\end{array}\right) \quad\left(\begin{array}{rr}
241 & 55 \\
-390 & -89
\end{array}\right) \\
& \left(\begin{array}{rr}
151 & 64 \\
-210 & -89
\end{array}\right) \quad\left(\begin{array}{rr}
-89 & -23 \\
120 & 31
\end{array}\right) \quad\left(\begin{array}{rr}
31 & 5 \\
-180 & -29
\end{array}\right)\left(\begin{array}{rr}
61 & 22 \\
-330 & -119
\end{array}\right) \\
& \left(\begin{array}{rr}
-119 & -32 \\
450 & 121
\end{array}\right) \quad\left(\begin{array}{rr}
-89 & -27 \\
300 & 91
\end{array}\right)\left(\begin{array}{rr}
31 & 10 \\
-90 & -29
\end{array}\right) \quad\left(\begin{array}{rr}
151 & 32 \\
-420 & -89
\end{array}\right) \\
& \left(\begin{array}{rr}
91 & 11 \\
-240 & -29
\end{array}\right) \quad\left(\begin{array}{rr}
-209 & -147 \\
300 & 211
\end{array}\right) \quad\left(\begin{array}{rr}
-149 & -22 \\
210 & 31
\end{array}\right)\left(\begin{array}{rr}
301 & 115 \\
-390 & -149
\end{array}\right) \\
& \left(\begin{array}{rr}
331 & 121 \\
-900 & -329
\end{array}\right) \quad\left(\begin{array}{rr}
-119 & -93 \\
270 & 211
\end{array}\right) \quad\left(\begin{array}{rr}
271 & 118 \\
-480 & -209
\end{array}\right)\left(\begin{array}{rr}
61 & 40 \\
-90 & -59
\end{array}\right) \\
& \left(\begin{array}{rr}
91 & 22 \\
-120 & -29
\end{array}\right) \quad\left(\begin{array}{rr}
-179 & -52 \\
210 & 61
\end{array}\right) \quad\left(\begin{array}{rr}
241 & 141 \\
-870 & -509
\end{array}\right)\left(\begin{array}{rr}
-389 & -151 \\
930 & 361
\end{array}\right) \\
& \left(\begin{array}{rr}
-389 & -114 \\
720 & 211
\end{array}\right)\left(\begin{array}{rr}
211 & 172 \\
-330 & -269
\end{array}\right)\left(\begin{array}{rr}
121 & 96 \\
-150 & -119
\end{array}\right)\left(\begin{array}{rr}
-59 & -27 \\
330 & 151
\end{array}\right) \\
& \left(\begin{array}{rr}
91 & 8 \\
-330 & -29
\end{array}\right) \quad\left(\begin{array}{rr}
-149 & -123 \\
510 & 421
\end{array}\right)\left(\begin{array}{rr}
361 & 212 \\
-1020 & -599
\end{array}\right)\left(\begin{array}{rr}
301 & 208 \\
-780 & -539
\end{array}\right) \\
& \left(\begin{array}{rr}
391 & 214 \\
-930 & -509
\end{array}\right) \quad\left(\begin{array}{rr}
121 & 13 \\
-270 & -29
\end{array}\right) \quad\left(\begin{array}{rr}
-299 & -82 \\
660 & 181
\end{array}\right)\left(\begin{array}{rr}
-269 & -48 \\
510 & 91
\end{array}\right)
\end{aligned}
$$




$$
\begin{aligned}
& \left(\begin{array}{rr}
331 & 212 \\
-420 & -269
\end{array}\right) \quad\left(\begin{array}{rr}
271 & 147 \\
-330 & -179
\end{array}\right) \quad\left(\begin{array}{rr}
-59 & -8 \\
450 & 61
\end{array}\right)\left(\begin{array}{rr}
-149 & -80 \\
840 & 451
\end{array}\right) \\
& \left(\begin{array}{rr}
121 & 67 \\
-540 & -299
\end{array}\right) \quad\left(\begin{array}{rr}
211 & 49 \\
-900 & -209
\end{array}\right)\left(\begin{array}{rr}
-269 & -35 \\
930 & 121
\end{array}\right)\left(\begin{array}{rr}
391 & 169 \\
-900 & -389
\end{array}\right) \\
& \left(\begin{array}{rr}
241 & 128 \\
-450 & -239
\end{array}\right) \quad\left(\begin{array}{rr}
-659 & -363 \\
1200 & 661
\end{array}\right)\left(\begin{array}{rr}
-119 & -27 \\
930 & 211
\end{array}\right)\left(\begin{array}{rr}
421 & 122 \\
-1860 & -539
\end{array}\right) \\
& \left(\begin{array}{rr}
-929 & -331 \\
1350 & 481
\end{array}\right)\left(\begin{array}{rr}
1861 & 661 \\
-2700 & -959
\end{array}\right)
\end{aligned}
$$

$\Gamma_{1}[45]$ is generated by 151 elements

$$
\begin{aligned}
& \left(\begin{array}{rr}
-1 & 0 \\
0 & -1
\end{array}\right)\left(\begin{array}{ll}
1 & 1 \\
0 & 1
\end{array}\right)\left(\begin{array}{rr}
181 & 49 \\
-495 & -134
\end{array}\right)\left(\begin{array}{rr}
91 & 20 \\
-405 & -89
\end{array}\right)\left(\begin{array}{rr}
-179 & -48 \\
675 & 181
\end{array}\right) \\
& \left(\begin{array}{rr}
-134 & -77 \\
315 & 181
\end{array}\right)\left(\begin{array}{rr}
271 & 98 \\
-495 & -179
\end{array}\right)\left(\begin{array}{rr}
226 & 125 \\
-405 & -224
\end{array}\right)\left(\begin{array}{rr}
91 & 66 \\
-495 & -359
\end{array}\right) \\
& \left(\begin{array}{rr}
181 & 76 \\
-855 & -359
\end{array}\right)\left(\begin{array}{rr}
-134 & -93 \\
585 & 406
\end{array}\right)\left(\begin{array}{rr}
136 & 111 \\
-495 & -404
\end{array}\right)\left(\begin{array}{rr}
-89 & -13 \\
315 & 46
\end{array}\right) \\
& \left(\begin{array}{rr}
-224 & -121 \\
585 & 316
\end{array}\right)\left(\begin{array}{rr}
91 & 36 \\
-225 & -89
\end{array}\right)\left(\begin{array}{rr}
136 & 81 \\
-225 & -134
\end{array}\right)\left(\begin{array}{rr}
361 & 229 \\
-495 & -314
\end{array}\right) \\
& \left(\begin{array}{rr}
-134 & -79 \\
765 & 451
\end{array}\right)\left(\begin{array}{rr}
46 & 9 \\
-225 & -44
\end{array}\right)\left(\begin{array}{rr}
-179 & -138 \\
585 & 451
\end{array}\right)\left(\begin{array}{rr}
136 & 19 \\
-315 & -44
\end{array}\right) \\
& \left(\begin{array}{rr}
181 & 80 \\
-405 & -179
\end{array}\right)\left(\begin{array}{rr}
-269 & -166 \\
585 & 361
\end{array}\right)\left(\begin{array}{rr}
-314 & -73 \\
585 & 136
\end{array}\right)\left(\begin{array}{rr}
631 & 164 \\
-1035 & -269
\end{array}\right) \\
& \left(\begin{array}{rr}
586 & 111 \\
-945 & -179
\end{array}\right)\left(\begin{array}{rr}
316 & 143 \\
-495 & -224
\end{array}\right)\left(\begin{array}{rr}
-494 & -175 \\
765 & 271
\end{array}\right)\left(\begin{array}{rr}
-629 & -398 \\
855 & 541
\end{array}\right) \\
& \left(\begin{array}{rr}
316 & 245 \\
-405 & -314
\end{array}\right)\left(\begin{array}{rr}
-674 & -391 \\
855 & 496
\end{array}\right)\left(\begin{array}{rr}
766 & 291 \\
-945 & -359
\end{array}\right)\left(\begin{array}{rr}
-179 & -78 \\
1035 & 451
\end{array}\right) \\
& \left(\begin{array}{rr}
-89 & -73 \\
495 & 406
\end{array}\right)\left(\begin{array}{rr}
181 & 53 \\
-765 & -224
\end{array}\right)\left(\begin{array}{rr}
361 & 221 \\
-1395 & -854
\end{array}\right)\left(\begin{array}{rr}
451 & 125 \\
-1620 & -449
\end{array}\right) \\
& \left(\begin{array}{rr}
361 & 227 \\
-1215 & -764
\end{array}\right)\left(\begin{array}{rr}
181 & 125 \\
-585 & -404
\end{array}\right)\left(\begin{array}{rr}
-359 & -94 \\
1035 & 271
\end{array}\right)\left(\begin{array}{rr}
451 & 317 \\
-1215 & -854
\end{array}\right)
\end{aligned}
$$


$\left(\begin{array}{rr}-494 & -307 \\ 1305 & 811\end{array}\right)\left(\begin{array}{rr}991 & 406 \\ -1755 & -719\end{array}\right)\left(\begin{array}{rr}1171 & 676 \\ -2025 & -1169\end{array}\right)\left(\begin{array}{rr}541 & 413 \\ -765 & -584\end{array}\right)$

$\left(\begin{array}{rr}-269 & -193 \\ 315 & 226\end{array}\right)\left(\begin{array}{rr}-89 & -12 \\ 675 & 91\end{array}\right)\left(\begin{array}{rr}361 & 64 \\ -2025 & -359\end{array}\right)\left(\begin{array}{rr}-179 & -95 \\ 765 & 406\end{array}\right)$

$\left(\begin{array}{rr}451 & 265 \\ -1530 & -899\end{array}\right)\left(\begin{array}{rr}-719 & -453 \\ 2430 & 1531\end{array}\right)\left(\begin{array}{rr}-269 & -32 \\ 765 & 91\end{array}\right)\left(\begin{array}{rr}-629 & -81 \\ 1755 & 226\end{array}\right)$

$\left(\begin{array}{rr}-404 & -65 \\ 1125 & 181\end{array}\right)\left(\begin{array}{rr}-899 & -633 \\ 2430 & 1711\end{array}\right) \quad\left(\begin{array}{rr}766 & 289 \\ -2025 & -764\end{array}\right) \quad\left(\begin{array}{rr}-944 & -275 \\ 2475 & 721\end{array}\right)$

$\left(\begin{array}{rr}361 & 34 \\ -945 & -89\end{array}\right)\left(\begin{array}{rr}631 & 245 \\ -1620 & -629\end{array}\right)\left(\begin{array}{rr}-314 & -259 \\ 765 & 631\end{array}\right)\left(\begin{array}{rr}631 & 445 \\ -1530 & -1079\end{array}\right)$

$\left(\begin{array}{rr}-764 & -429 \\ 1845 & 1036\end{array}\right)\left(\begin{array}{rr}-809 & -245 \\ 1935 & 586\end{array}\right)\left(\begin{array}{rr}-809 & -474 \\ 1845 & 1081\end{array}\right)$

$\left(\begin{array}{rr}1171 & 845 \\ -1620 & -1169\end{array}\right)\left(\begin{array}{rr}-854 & -479 \\ 1125 & 631\end{array}\right)\left(\begin{array}{rr}811 & 105 \\ -1035 & -134\end{array}\right)\left(\begin{array}{rr}-89 & -61 \\ 855 & 586\end{array}\right)$

$\left(\begin{array}{rc}46 & 5 \\ -405 & -44\end{array}\right)\left(\begin{array}{rr}-179 & -69 \\ 1170 & 451\end{array}\right)\left(\begin{array}{rr}271 & 174 \\ -1260 & -809\end{array}\right)\left(\begin{array}{rr}-449 & -254 \\ 2070 & 1171\end{array}\right)$

$\left(\begin{array}{rr}1306 & 547 \\ -4725 & -1979\end{array}\right)\left(\begin{array}{rr}271 & 71 \\ -855 & -224\end{array}\right)\left(\begin{array}{rr}721 & 256 \\ -2025 & -719\end{array}\right)\left(\begin{array}{rr}1711 & 992 \\ -4500 & -2609\end{array}\right)$

$\left(\begin{array}{rr}1621 & 474 \\ -3690 & -1079\end{array}\right)\left(\begin{array}{rr}721 & 554 \\ -1170 & -899\end{array}\right)\left(\begin{array}{rr}1216 & 367 \\ -1935 & -584\end{array}\right)\left(\begin{array}{rr}-1169 & -739 \\ 1710 & 1081\end{array}\right)$

$\left(\begin{array}{rr}-989 & -557 \\ 1440 & 811\end{array}\right)\left(\begin{array}{rr}1396 & 961 \\ -2025 & -1394\end{array}\right)\left(\begin{array}{rr}991 & 287 \\ -1395 & -404\end{array}\right)\left(\begin{array}{rr}-1394 & -487 \\ 1935 & 676\end{array}\right)$

$\left(\begin{array}{rr}721 & 113 \\ -855 & -134\end{array}\right)\left(\begin{array}{rr}271 & 154 \\ -315 & -179\end{array}\right)\left(\begin{array}{rr}-44 & -17 \\ 585 & 226\end{array}\right)\left(\begin{array}{rr}361 & 211 \\ -2385 & -1394\end{array}\right)$

$\left(\begin{array}{rr}316 & 49 \\ -2025 & -314\end{array}\right)\left(\begin{array}{rr}271 & 171 \\ -1710 & -1079\end{array}\right)\left(\begin{array}{rr}-224 & -195 \\ 1035 & 901\end{array}\right)\left(\begin{array}{rr}1621 & 339 \\ -5805 & -1214\end{array}\right)$

$\left(\begin{array}{rr}586 & 169 \\ -2025 & -584\end{array}\right)\left(\begin{array}{rr}1216 & 745 \\ -4185 & -2564\end{array}\right)\left(\begin{array}{rr}-494 & -85 \\ 1575 & 271\end{array}\right)\left(\begin{array}{rr}631 & 531 \\ -1710 & -1439\end{array}\right)$

$\left(\begin{array}{rr}-1529 & -967 \\ 3915 & 2476\end{array}\right)\left(\begin{array}{rr}-1889 & -409 \\ 3330 & 721\end{array}\right)\left(\begin{array}{rr}991 & 521 \\ -1710 & -899\end{array}\right)\left(\begin{array}{rr}2746 & 1987 \\ -4725 & -3419\end{array}\right)$

$\left(\begin{array}{rr}-1754 & -1231 \\ 3015 & 2116\end{array}\right)\left(\begin{array}{rr}3961 & 1531 \\ -6750 & -2609\end{array}\right)\left(\begin{array}{rr}4141 & 1711 \\ -6750 & -2789\end{array}\right)$ 


$$
\begin{aligned}
& \left(\begin{array}{rr}
-4814 & -1865 \\
7785 & 3016
\end{array}\right)\left(\begin{array}{rr}
-1439 & -311 \\
2295 & 496
\end{array}\right)\left(\begin{array}{rr}
-2969 & -1096 \\
4635 & 1711
\end{array}\right) \\
& \left(\begin{array}{rr}
946 & 335 \\
-1395 & -494
\end{array}\right)\left(\begin{array}{rr}
-1349 & -785 \\
1935 & 1126
\end{array}\right)\left(\begin{array}{rr}
631 & 441 \\
-900 & -629
\end{array}\right)\left(\begin{array}{rr}
4141 & 1485 \\
-5895 & -2114
\end{array}\right) \\
& \left(\begin{array}{rr}
-1754 & -1085 \\
2475 & 1531
\end{array}\right)\left(\begin{array}{rr}
1846 & 647 \\
-2565 & -899
\end{array}\right)\left(\begin{array}{rr}
2791 & 585 \\
-3645 & -764
\end{array}\right) \\
& \left(\begin{array}{rr}
3241 & 1999 \\
-4230 & -2609
\end{array}\right)\left(\begin{array}{rr}
-944 & -605 \\
1125 & 721
\end{array}\right)\left(\begin{array}{rr}
-89 & -62 \\
1035 & 721
\end{array}\right)\left(\begin{array}{rr}
-224 & -47 \\
1935 & 406
\end{array}\right) \\
& \left(\begin{array}{rr}
766 & 271 \\
-5085 & -1799
\end{array}\right)\left(\begin{array}{rr}
1441 & 596 \\
-5220 & -2159
\end{array}\right)\left(\begin{array}{rr}
-2474 & -1517 \\
8955 & 5491
\end{array}\right) \\
& \left(\begin{array}{rr}
-629 & -428 \\
2250 & 1531
\end{array}\right)\left(\begin{array}{rr}
-2564 & -947 \\
8775 & 3241
\end{array}\right)\left(\begin{array}{rr}
1621 & 1150 \\
-4185 & -2969
\end{array}\right)\left(\begin{array}{rr}
1936 & 331 \\
-4995 & -854
\end{array}\right) \\
& \left(\begin{array}{rr}
-1439 & -505 \\
3465 & 1216
\end{array}\right)\left(\begin{array}{rr}
-3959 & -2497 \\
7065 & 4456
\end{array}\right)\left(\begin{array}{rr}
-5759 & -3527 \\
10215 & 6256
\end{array}\right) \\
& \left(\begin{array}{rr}
3241 & 1254 \\
-5580 & -2159
\end{array}\right)\left(\begin{array}{rr}
4186 & 1737 \\
-7155 & -2969
\end{array}\right)\left(\begin{array}{rr}
5041 & 2929 \\
-8595 & -4994
\end{array}\right) \\
& \left(\begin{array}{rr}
4006 & 1215 \\
-6525 & -1979
\end{array}\right)\left(\begin{array}{rr}
1576 & 1133 \\
-2565 & -1844
\end{array}\right)\left(\begin{array}{rr}
-4949 & -1502 \\
8010 & 2431
\end{array}\right) \\
& \left(\begin{array}{rr}
2161 & 657 \\
-3105 & -944
\end{array}\right)\left(\begin{array}{rr}
-1709 & -958 \\
8190 & 4591
\end{array}\right)\left(\begin{array}{rr}
2791 & 815 \\
-8010 & -2339
\end{array}\right)\left(\begin{array}{rr}
361 & 188 \\
-1035 & -539
\end{array}\right) \\
& \left(\begin{array}{rr}
856 & 145 \\
-2385 & -404
\end{array}\right)\left(\begin{array}{rr}
3916 & 2297 \\
-10125 & -5939
\end{array}\right)\left(\begin{array}{rr}
12511 & 2700 \\
-20430 & -4409
\end{array}\right) \\
& \left(\begin{array}{rr}
-1349 & -477 \\
1530 & 541
\end{array}\right)\left(\begin{array}{rr}
856 & 529 \\
-945 & -584
\end{array}\right)\left(\begin{array}{rc}
2206 & 379 \\
-4185 & -719
\end{array}\right)\left(\begin{array}{rr}
-1214 & -429 \\
2295 & 811
\end{array}\right) \\
& \left(\begin{array}{rr}
-1799 & -1112 \\
10485 & 6481
\end{array}\right)\left(\begin{array}{rr}
7111 & 1485 \\
-24345 & -5084
\end{array}\right)\left(\begin{array}{rr}
-3419 & -583 \\
15840 & 2701
\end{array}\right) \\
& \left(\begin{array}{rr}
5536 & 1681 \\
-18225 & -5534
\end{array}\right)\left(\begin{array}{rr}
7561 & 3136 \\
-18225 & -7559
\end{array}\right)\left(\begin{array}{rr}
3106 & 529 \\
-18225 & -3104
\end{array}\right) \\
& \left(\begin{array}{rr}
10576 & 2209 \\
-50625 & -10574
\end{array}\right)
\end{aligned}
$$

These generating sets are, perhaps surprisingly, minimal or close to minimal. A lower bound for the number of generators for $\Gamma_{1}[N]$ is $2+$ integer part of $\left[\Gamma: \Gamma_{1}[N]\right] / 6$ (this can be deduced from Theorem 
VIII.7 of Newman $\left.{ }^{(18)}\right)$. So we have minimal generating sets for $\Gamma_{1}[5]$ and $\Gamma_{1}[15]$. Using programs for calculating presentations of subgroups of finite index in finitely presented groups and for transforming finite presentation (see Havas et al. ${ }^{(19)}$ ), it is possible to find relations among the generators in the generating sets for $\Gamma_{1}[30]$ and $\Gamma_{1}[45]$. These relations can be used to get minimal generating sets.

Since $\Gamma_{1}[15]$ is a subgroup of index 8 in $\Gamma_{1}[5]$, another approach is to start from a generating set for $\Gamma_{1}[5]$ and proceed similarly. The resulting generating set is less pleasing, since larger entries arise in the matrices.

\section{NOTE ADDED IN PROOF}

After this paper was submitted, the algorithm in Appendix $\mathrm{C}$ was implemented using PASCAL. Copies are available by writing to L. Grove.

The polynomial (1.2) has been computed in "Equation of State and Isothermal Compressibility for the Hard Hexagon Model in the Disordered Regime" by M. P. Richey and C. A. Tracy, submitted to J. Phys. A: Math. Gen.

The exponent 5/6 appearing in Eq. (4.8) has also been computed by A. Baram and M. Luban, "Universality of the Cluster Integrals of Repulsive Systems," to appear in Physical Review A.

\section{ACKNOWLEDGMENTS}

It is a pleasure to thank Profs. Rodney J. Baxter, John D. Fay, Michael E. Fisher, Charles M. Newman, and Thomas Shemanske for useful comments and suggestions. The first author wishes to thank Prof. Mario Rasetti for the invitation to visit the ISI, where part of this work was done.

This work was supported in part by National Science Foundation grant DMS 84-21141 (to C.A.T.).

\section{REFERENCES}

1. R. J. Baxter, J. Phys. A: Math. Gen. 13:L61 (1980).

2. R. J. Baxter, J. Stat. Phys. 26:427 (1981).

3. R. J. Baxter, Exactly Solved Models in Statistical Mechanics (Academic Press, London, 1982).

4. R. J. Baxter and P. A. Pearce, J. Phys. A.: Math. Gen. 15:897 (1982).

5. R. J. Baxter and P. A. Pearce, J. Phys. A.: Math. Gen. 16:2239 (1983).

6. P. A. Pearce and R. J. Baxter, J. Phys. A.: Math. Gen. 17:2095 (1984).

7. R. J. Baxter, in Integrable Systems in Statistical Mechanics, G. M. D'Ariano, A. Montorsi, and M. G. Rasetti, eds. (World Scientific, Singapore, 1985). 
8. P. A. Pearce, Sci. Prog. Oxf. 68:189 (1983).

9. J. Groeneveld, Phys. Lett. 3:50 (1962).

10. D. S. Gaunt, J. Chem. Phys. 46:3237 (1967).

11. B. Schoeneberg, Elliptic Modular Functions (Springer-Verlag, Berlin, 1974).

12. F. Klein and R. Fricke, Vorlesungen über die Theorie der Elliptischen Modulfunctionen, Vol. I (B. G. Teubner, Leipzig, 1890).

13. A. Erdelyi, Higher Transcendental Functions, Vol. 3 (McGraw-Hill, New York, 1955).

14. H. Maass, Modular Functions of One Complex Variable (Springer-Verlag, Berlin, 1983).

15. A. P. Ogg, in Analytic Number Theory, Proceedings of Symposium in Pure Mathematics, Vol. XXIV (AMS, Providence, 1973), p. 221.

16. D. J. S. Robinson, A Course in the Theory of Groups (Springer-Verlag, New York, 1982).

17. J. J. Cannon, in Computational Group Theory, M. D. Atkinson, ed. (Academic Press, London, 1984).

18. M. Newman, Integral Matrices (Academic Press, New York, 1972).

19. G. Havas, P. E. Kenne, J. S. Richardson, and E. F. Robertson in Computational Group Theory, M. D. Atkinson, ed. (Academic Press, London, 1984). 